\title{
THE STRUCTURE OF TENSOR PRODUCTS OF SEMILATTICES WITH ZERO
}

\author{
BY
}

\author{
G. GRÄTZER, H. LAKSER AND R. QUACKENBUSH ${ }^{1}$
}

\begin{abstract}
If $A$ and $B$ are finite lattices, then the tensor product $C$ of $A$ and $B$ in the category of join semilattices with zero is a lattice again. The main result of this paper is the description of the congruence lattice of $C$ as the free product (in the category of bounded distributive lattices) of the congruence lattice of $A$ and the congruence lattice of $B$. This provides us with a method of constructing finite subdirectly irreducible (resp., simple) lattices: if $A$ and $B$ are finite subdirectly irreducible (resp., simple) lattices then so is their tensor product. Another application is a result of E. T. Schmidt describing the congruence lattice of a bounded distributive extension of $M_{3}$.
\end{abstract}

1. Introduction. Tensor products of semilattices have been studied by J. Anderson and N. Kimura [1], G. Fraser [2]-[5], and Z. Shmuely [9]. In this paper we deal with join semilattices with zero. In certain cases the tensor product of two semilattices with zero is a lattice; thus, we can investigate the lattice of lattice congruences of the tensor product. Our main result is a representation theorem: The congruence lattice of such a tensor product is a tensor product of the congruence lattices.

More precisely, we are working in the category $S_{0}$, whose objects are join semilattices with zero and whose morphisms are the zero-preserving join homomorphisms. Similarly, $S_{1}$ is the category of meet semilattices with unit whose morphisms are the unit-preserving meet homomorphisms. If $A \in S_{0}$ is a lattice, then $\operatorname{Con}_{L}(A)$ denotes the lattice of lattice congruences of $A$. Let $\otimes$ denote the tensor product in $S_{0}$. Our theorem then states that under suitable conditions on $A, B \in$ $S_{0}$,

$$
\operatorname{Con}_{L}(A \otimes B) \simeq \operatorname{Con}_{L}(A) \otimes \operatorname{Con}_{L}(B) .
$$

This result has two interesting consequences. $A \otimes B$ is a finite simple (respectively, subdirectly irreducible) lattice iff both $A$ and $B$ are finite simple (respectively, subdirectly irreducible) lattices.

Note that, in general, the tensor product of $A$ and $B$ in $S_{0}$ is not isomorphic to the tensor product of $A$ and $B$ in $S_{1}$ even though their congruence lattices are isomorphic.

Received by the editors July 16, 1979.

1980 Mathematics Subject Classification. Primary 06B05, 06B10.

Key words and phrases. Semilattice, lattice, tensor product, congruence lattice, simple, subdirectly irreducible.

${ }^{1}$ The research of the authors was supported by the Natural Sciences and Engineering Research Council of Canada. 
We next describe how we came to the main result. In Schmidt [8], the lattice $M_{3}[D]$ is discussed. For a finite distributive lattice $d, M_{3}[D]$ denotes the lattice of all isotone maps from the poset of join irreducible elements of $D$ into $M_{3}$. This is isomorphic to $\operatorname{Hom}_{0}\left(D, M_{3}\right)$ the lattice of all zero preserving join homomorphisms of $D$ into $M_{3}$. E. T. Schmidt shows that $M_{3}\left[D^{d}\right]$ (where $D^{d}$ is the dual of $D$ ) is isomorphic to the subposet of $D^{3}$ consisting of all ordered triples $(a, b, c)$ such that $a \wedge b=a \wedge c=b \wedge c$. But this latter poset is isomorphic to $\operatorname{Hom}_{1}\left(M_{3}, D\right)$, the poset (in fact, lattice) of all unit-preserving meet homomorphisms of $M_{3}$ into $D$. Thus Schmidt's result can be stated as follows:

$$
\operatorname{Hom}_{0}\left(D^{d}, M_{3}\right) \simeq \operatorname{Hom}_{1}\left(M_{3}, D\right) \text {. }
$$

Moreover, he proves that the congruence lattice of $M_{3}[D]$ is isomorphic to the congruence lattice of $D$.

We will generalize Schmidt's result and show that it readily follows from our results. However, we must proceed with some care due to the numerous dualities present. For $A \in S_{i}$, let $A^{d}$ be the dual of $A$. Note that $M_{3} \simeq M_{3}^{d}$ and that although in general $D \nsim D^{d}$, they have dually isomorphic posets of join irreducible elements. We will show that

$$
A \otimes B \simeq\left[\operatorname{Hom}_{0}(A, B)\right]^{d} .
$$

To obtain Schmidt's result, let $J(D)$ (respectively, $M(D)$ ) be the poset of join (respectively, meet) irreducible elements of a finite $D \in D_{0,1}$; it is well known that $J(D) \simeq M(D)$. For posets $P$ and $Q$, let $Q[P]$ be the poset of isotone maps from $P$ to $Q$. Since $D$ is a finite distributive lattice,

$$
\operatorname{Hom}_{0}\left(D^{d}, M_{3}\right) \simeq M_{3}\left[J\left(D^{d}\right)\right] \simeq M_{3}\left[M\left(D^{d}\right)\right] \simeq \operatorname{Hom}_{1}\left(D^{d}, M_{3}\right) .
$$

But the commutativity of the tensor product in $S_{1}$ now tells us that

$$
\operatorname{Hom}_{1}\left(D^{d}, M_{3}\right) \simeq \operatorname{Hom}_{1}\left(M_{3}, D^{d}\right) \simeq \operatorname{Hom}_{1}\left(M_{3}, D\right)
$$

which is Schmidt's result.

For notation and concepts not defined in this paper, see [7].

2. The tensor product. Let $A, B, C \in S_{0}$; a map $f: A \times B \rightarrow C$ is a bimorphism if for each $a \in A$ and $b \in B$, we have $f(a,-) \in \operatorname{Hom}_{0}(B, C)$ and $f(-, b) \in$ $\operatorname{Hom}_{0}(A, C)$. Notice that if $f: A \times B \rightarrow C$ is a bimorphism, then for all $a \in A$ and $b \in B, f(a, 0)=f(0, b)=0$.

We say that $A \otimes B \in S_{0}$ is a tensor product of $A$ and $B$ in $S_{0}$ if there is a bimorphism $f: A \times B \rightarrow A \otimes B$ such that

(i) $f(A \times B)$ generates $A \otimes B$;

(ii) for any bimorphism $g: A \times B \rightarrow C$, there is an $h \in \operatorname{Hom}_{0}(A \otimes b, C)$ such that $g=h f$.

Let $A \circ B$ be the direct product of the posets $A-\{0\}$ and $B-\{0\}$; we make $A \circ B$ into a partial join semilattice as follows: If $(a, b)$ and $\left(a^{\prime}, b^{\prime}\right)$ are comparable or if $a=a^{\prime}$ or if $b=b^{\prime}$, then $(a, b) \vee\left(a^{\prime}, b^{\prime}\right)$ is defined and equals $\left(a \vee a^{\prime}\right.$, $\left.b \vee b^{\prime}\right)$; otherwise $(a, b) \vee\left(a^{\prime}, b^{\prime}\right)$ is undefined. Let $F_{0}(A \circ B)$ be the join semilattice with zero freely generated by $A \circ B$ (in particular, a zero is added even if 
$A \circ B$ has a least element). It is easily seen that $F_{0}(A \circ B)$ is a tensor product of $A$ and $B$. This implies that the tensor product is commutative and associative. We will denote the elements of $A \circ B$ by $a \otimes b$; if $a=0$ or $b=0$, then $a \otimes b$ denotes 0 .

If $A$ and $B$ are finite, $F_{0}(A \circ B)$ is isomorphic to the lattice of all ideals of $A \circ B$; recall that a subset $I$ of $A \circ B$ is an ideal provided:

(i) if $(a, b) \in I$ and $\left(a^{\prime}, b^{\prime}\right) \leqslant(a, b)$, then $\left(a^{\prime}, b^{\prime}\right) \in I$;

(ii) if $(a, b),\left(a^{\prime}, b^{\prime}\right) \in I$ and $(a, b) \vee\left(a^{\prime}, b^{\prime}\right)$ is defined, then $(a, b) \vee\left(a^{\prime}, b^{\prime}\right) \in I$.

Note that the empty set is an ideal.

G. Fraser [4] solved the word problem for the tensor product in the category of join semilattices; we state the analogous result for lattices in $S_{0}$.

Theorem 2.1 (G. Fraser [4]). Let $A, B \in S_{0}$ be lattices. Let $a, a_{1}, \ldots, a_{n} \in A$ $-\{0\}$ and let $b, b_{1}, \ldots, b_{n} \in B-\{0\}$. Then $a \otimes b \leqslant \bigvee\left\{a_{i} \otimes b_{i} \mid 1<i<n\right\}$ iff there is an $n$-ary lattice polynomial $p$ such that $a \leqslant p\left(a_{1}, \ldots, a_{n}\right)$ and $b<$ $p^{d}\left(b_{1}, \ldots, b_{n}\right)$, where $p^{d}$ is the dual of $p$.

Corollary 2.2 (G. Fraser [4]). Let $A, B \in S_{0}$ be lattices. Let $a, a_{1}, a_{2} \in A-$ $\{0\}$ and let $b, b_{1}, b_{2} \in B-\{0\}$. Then $a \otimes b \leqslant\left(a_{1} \otimes b_{1}\right) \vee\left(a_{2} \otimes b_{2}\right)$ iff one of the following four conditions hold.

(i) $a \leqslant a_{1}$ and $b \leqslant b_{1}$;

(ii) $a \leqslant a_{2}$ and $b \leqslant b_{2}$;

(iii) $a \leqslant a_{1} \vee a_{2}$ and $b \leqslant b_{1} \wedge b_{2}$;

(iv) $a \leqslant a_{1} \wedge a_{2}$ and $b \leqslant b_{1} \vee b_{2}$.

Corollary 2.3. Let $A, B \in S_{0}$ be lattices. Let $a, a_{1}, a_{2} \in A-\{0\}$ and let $b, b_{1}, b_{2} \in B-\{0\}$. Let $a_{1} \leqslant a_{2}$ and $b_{2} \leqslant b_{1}$, or let $a_{2} \leqslant a_{1}$ and $b_{1} \leqslant b_{2}$; then $a \otimes b \leqslant\left(a_{1} \otimes b_{1}\right) \vee\left(a_{2} \otimes b_{2}\right)$ iff $a \otimes b \leqslant a_{1} \otimes b_{1}$ or $a \otimes b \leqslant a_{2} \otimes b_{2}$.

This is the first of two key observations; it tells us that, under the conditions stated the ideal of $A \circ B$ generated by $\left\{a_{1} \otimes b_{1}, a_{2} \otimes b_{2}\right\}$ is the union of the principal ideals generated by $a_{1} \otimes b_{1}$ and $a_{2} \otimes b_{2}$. The second key observation follows.

Corollary 2.4. Let $A$ and $B$ be lattices. For $a, a_{i}, c_{j} \in A-\{0\}$ and $b, b_{i}, d_{j} \in B$ $-\{0\}, a \otimes b \leqslant \bigvee\left\{a_{i} \otimes b_{i} \mid 1 \leqslant i \leqslant n\right\}$ and $a \otimes b \leqslant \bigvee\left\{c_{j} \otimes d_{j} \mid 1<j<m\right\}$ iff there are polynomials $p$ and $q$ such that $a \leqslant p\left(a_{1}, \ldots, a_{n}\right) \wedge q\left(c_{1}, \ldots, c_{m}\right)$ and $b \leqslant p^{d}\left(b_{1}, \ldots, b_{n}\right) \wedge q^{d}\left(d_{1}, \ldots, d_{m}\right)$.

Corollary 2.4 will be used to describe meets in $A \otimes B$, provided that it is a lattice.

Let $C_{2}$ denote the 2-element semilattice. Let $A, B \in S_{0}$ be finite. It is easily seen that $\operatorname{Hom}_{0}\left(A, C_{2}\right) \simeq A^{d}$. Since $\operatorname{Hom}_{0}(A, B) \in S_{0}$,

$$
\operatorname{Hom}_{0}(A \otimes B, C) \simeq \operatorname{Hom}_{0}\left(A, \operatorname{Hom}_{0}(B, C)\right)
$$

Thus

$$
(A \otimes B)^{d} \simeq \operatorname{Hom}_{0}\left(A \otimes B, C_{2}\right) \simeq \operatorname{Hom}_{0}\left(A, B^{d}\right)
$$


Theorem 2.5 (J. Anderson ANd N. Kimura [1]). Let $A, B \in S_{0}$ be finite; then $A \otimes B \simeq\left(\operatorname{Hom}_{0}\left(A, B^{d}\right)\right)^{d}$.

Of course, we can define the tensor product in $S_{1}$ analogously and obtain analogous results.

Let $A, B \in S_{0}$ be lattices; when is $A \otimes B$ a lattice? By 2.4, if $A \otimes B$ is a lattice, then

$$
\bigvee\left\{a_{i} \otimes b_{i} \mid 1 \leqslant i \leqslant n\right\} \wedge \bigvee\left\{c_{j} \otimes d_{j} \mid 1 \leqslant j \leqslant m\right\}
$$

is the join of all elements of the form

$$
\left(p\left(a_{1}, \ldots, a_{n}\right) \wedge q\left(c_{1}, \ldots, c_{m}\right)\right) \otimes\left(p^{d}\left(b_{1}, \ldots, b_{n}\right) \wedge q^{d}\left(d_{1}, \ldots, d_{m}\right)\right),
$$

where $p$ and $q$ are lattice polynomials. This join will exist exactly when it is equal to the join of finitely many of its components. We can guarantee that the join exists by requiring that $A$ be locally finite and that $B$ be $A$-lower bounded. To define this latter condition, let $F_{C}$ be the free lattice over $C$ on countably many free generators $x_{1}, \ldots$, and let $F$ be the free lattice on countably many free generators $x_{1}, \ldots$; let $\rho_{C}: F \rightarrow F_{C}$ be induced by mapping $x_{i}$ to $x_{i}$ for all $i$. We say that $B$ is $A$-lower bounded if for every $p \in F_{A}, \rho_{B}\left(\rho_{A}^{-1}(p)\right)$ contains a least element (denoted by $p_{1}$ ).

Theorem 2.6. Let $A, B \in S_{0}$ be lattices. If $A$ is locally finite and if $B$ is $A$-lower bounded, then $A \otimes B$ is a lattice.

Proof. From 2.4 and our assumption that $B$ is $A$-lower bounded we see that

$$
\bigvee\left\{a_{i} \otimes b_{i} \mid 1 \leqslant i \leqslant n\right\} \wedge \bigvee\left\{c_{j} \otimes d_{j} \mid 1 \leqslant j \leqslant m\right\}
$$

is the join of all elements of the form

$$
\left(p\left(a_{1}, \ldots, a_{n}\right) \wedge q\left(c_{1}, \ldots, c_{m}\right)\right) \otimes\left(p_{1}^{d}\left(b_{1}, \ldots, b_{n}\right) \wedge q_{1}^{d}\left(d_{1}, \ldots, d_{m}\right)\right)
$$

where $p$ and $q \in F_{A}$; since $A$ is locally finite, this is indeed a finite join.

Corollary 2.7. If $A, B \in S_{0}$ are locally finite lattices, then $A \otimes B$ is a locally finite lattice.

Corollary 2.8. If $A$ is a distributive lattice, then $A \otimes B$ is a lattice.

Proof. Let $A$ be distributive; thus $A$ is locally finite. If $p \in F_{A}$, then the least element in $\rho_{A}^{-1}(p)$ is the disjunctive normal form of the lattice polynomials in $\rho_{A}^{-1}(p)$. Thus for any $B, \rho_{B}\left(\rho_{A}^{-1}(p)\right)$ has a least element; that is, $B$ is $A$-lower bounded.

3. Proof of the main result. In this section let $A$ be a finite lattice and let $B$ be an $A$-lower bounded lattice with 0 . As a first step, we embed $\operatorname{Con}_{L}(A)$ and $\operatorname{Con}_{L}(B)$ into $\operatorname{Con}_{L}(A \otimes B)$.

Let $C$ and $D$ be lattices with 0 such that $C \otimes D$ is a lattice. For $\phi \in \operatorname{Con}_{L}(C)$ and $\psi \in \operatorname{Con}_{L}(D)$, let $\phi^{*}$ be the lattice congruence on $C \otimes D$ generated by $\left\{\left(c_{1} \otimes d, c_{2} \otimes d\right) \mid\left(c_{1}, c_{2}\right) \in \phi, d \in D\right\}$, and let $\psi^{*}$ be the lattice congruence on $C \otimes D$ generated by $\left\{\left(c \otimes d_{1}, c \otimes d_{2}\right) \mid c \in C,\left(d_{1}, d_{2}\right) \in \psi\right\}$. 
LEMMA 3.1. The following isomorphism holds.

$$
(C \otimes D) / \phi^{*} \simeq(C / \phi) \otimes D \text {. }
$$

Furthermore, $\phi^{*}$ is the semilattice congruence generated by $\left\{\left(c_{1} \otimes d, c_{2} \otimes d\right) \mid\left(c_{1}, c_{2}\right)\right.$ $\in \phi, d \in D\}$. Also, the corresponding statement holds for $\psi^{*}$.

Proof. Let $f_{\phi}(c \otimes d)=[c] \phi \otimes d ; f_{\phi}$ extends to a homomorphism from $C \otimes D$ onto $(C / \phi) \otimes D$; this homomorphism will also be denoted by $f_{\phi}$. Clearly, $\operatorname{Ker}\left(f_{\phi}\right)$ is the semilattice congruence generated by $\left\{\left(c_{1} \otimes d, c_{2} \otimes d\right) \mid\left(c_{1}, c_{2}\right) \in \phi, d \in D\right\}$. To show that this establishes the required isomorphism, it suffices to show that $f_{\phi}$ preserves meets. Let

$$
x=\bigvee\left\{a_{i} \otimes b_{i} \mid 1 \leqslant i \leqslant n\right\}, \quad y=\bigvee\left\{c_{j} \otimes d_{j} \mid 1 \leqslant j \leqslant m\right\} .
$$

Since $f_{\phi}$ is isotone, we need only show that

$$
f_{\phi}(x) \wedge f_{\phi}(y) \leqslant f_{\phi}(x \wedge y) \text {. }
$$

In order to do this, let

$$
\begin{aligned}
{[c] \phi \otimes d \leqslant f_{\phi}(x) \wedge f_{\phi}(y)=} & \bigvee\left\{\left[a_{i}\right] \phi \otimes b_{i} \mid 1 \leqslant i \leqslant n\right\} \\
& \wedge \bigvee\left\{\left[c_{j}\right] \phi \otimes d_{j} \mid 1 \leqslant j \leqslant m\right\} .
\end{aligned}
$$

By 2.4, there are lattice polynomials $p$ and $q$ such that

$$
[c] \phi \leqslant p\left(\left[a_{1}\right] \phi, \ldots,\left[a_{n}\right] \phi\right) \wedge q\left(\left[c_{1}\right] \phi, \ldots,\left[c_{m}\right] \phi\right)
$$

and

$$
d \leqslant p^{d}\left(b_{1}, \ldots, b_{n}\right) \wedge q^{d}\left(d_{1}, \ldots, d_{m}\right) .
$$

Since $\phi$ is a lattice congruence, the former condition reduces to

$$
[c] \phi \leqslant\left[p\left(a_{1}, \ldots, a_{n}\right) \wedge q\left(c_{1}, \ldots, c_{m}\right)\right] \phi .
$$

Thus, without loss of generality, we may assume that

$$
c \leqslant p\left(a_{1}, \ldots, a_{n}\right) \wedge q\left(c_{1}, \ldots, c_{m}\right) .
$$

Hence $c \otimes d \leqslant x \wedge y$, and so

$$
[c] \phi \otimes d=f_{\phi}(c \otimes d) \leqslant f_{\phi}(x \wedge y)
$$

as desired.

Our next step is to describe principal lattice congruences on $A \otimes B$. As usual, let $\theta(f, g)$ denote the smallest lattice congruence on $A \otimes B$ containing $(f, g)$; we may assume that $f \geqslant g$. For $a \in A, h \in A \otimes B$, let

$$
a_{h}=\bigvee\{b \mid b \in B \text { and } a \otimes b \leqslant h\} ;
$$

since $A$ is finite and $B$ is $A$-lower bounded, $a_{h}$ exists.

LEMMA 3.2. $\left(a \vee a^{\prime}\right)_{h}=a_{h} \wedge a_{h}^{\prime}$.

Proof. It is easily seen that $\left(a \vee a^{\prime}\right)_{h} \leqslant a_{h} \wedge a_{h}^{\prime}$. Conversely, if $\left(a \otimes a_{h}\right)<h$ and $\left(a^{\prime} \otimes a_{h}^{\prime}\right) \leqslant h$, then $\left(a \otimes a_{h}\right) \vee\left(a^{\prime} \otimes a_{h}^{\prime}\right) \leqslant h$. By 2.2 ,

$$
\left(a \vee a^{\prime} \otimes a_{h} \wedge a_{h}^{\prime}\right) \leqslant\left(a \otimes a_{h}\right) \vee\left(a^{\prime} \otimes a_{h}^{\prime}\right),
$$

implying that $a_{h} \wedge a_{h}^{\prime} \leqslant\left(a \vee a^{\prime}\right)_{h}$. 
Thus, every $h \in A \otimes B$ can be written as $h=\bigvee\left\{a \otimes a_{h} \mid a \in J(A)\right\}$.

Whenever $a \in J(A)$, let $a_{+}$denote the unique element of $A$ covered by $a$.

LEMMA 3.3. For any $a \in J(A)$ and any $h \in A \otimes B$,

$$
(h \wedge(a \otimes 1)) \vee\left(a_{+} \otimes 1\right)=\left(a \otimes a_{h}\right) \vee\left(a_{+} \otimes 1\right)
$$

Proof. Clearly,

$$
(h \wedge(a \otimes 1)) \vee\left(a_{+} \otimes 1\right) \geqslant\left(a \otimes a_{h}\right) \vee\left(a_{+} \otimes 1\right)
$$

and $a_{+} \otimes 1 \leqslant\left(a \otimes a_{h}\right) \vee\left(a_{+} \otimes 1\right)$. Thus we need only show that

$$
h \wedge(a \otimes 1) \leqslant\left(a \otimes a_{h}\right) \vee\left(a_{+} \otimes 1\right) \text {. }
$$

Let $a^{\prime} \otimes b^{\prime} \leqslant h \wedge(a \otimes 1)$; then $a^{\prime} \otimes b^{\prime} \leqslant h$ and $a^{\prime} \otimes b^{\prime}<a \otimes 1$. This latter condition means that $a^{\prime} \leqslant a$. If $a^{\prime}<a$, then $a^{\prime} \leqslant a_{+}$and, hence, $a^{\prime} \otimes b^{\prime} \leqslant a_{+} \otimes 1$. If $a^{\prime}=a$, then $a^{\prime} \otimes b^{\prime} \leqslant h$ means that $a^{\prime} \otimes b^{\prime} \leqslant a \otimes a_{h}$. Thus

$$
h \wedge(a \otimes 1) \leqslant\left(a \otimes a_{h}\right) \vee\left(a_{+} \otimes 1\right) .
$$

COROLlary 3.4. Let $a \in J(A)$; then the following congruence holds.

$$
\left(a \otimes a_{f}\right) \vee\left(a_{+} \otimes 1\right) \equiv\left(a \otimes a_{g}\right) \vee\left(a_{+} \otimes 1\right)(\theta(f, g))
$$

LEMmA 3.5. Let $a \in J(A)$ and let

$$
\theta_{f, g}=\bigvee\left\{\theta\left(\left(a \otimes a_{f}\right) \vee\left(a_{+} \otimes 1\right),\left(a \otimes a_{g}\right) \vee\left(a_{+} \otimes 1\right)\right) \mid a \in J(A)\right\} ;
$$

then $f \equiv g\left(\theta_{f, g}\right)$.

Proof. For $a \in J(A)$, let

$$
f_{a}=\left(f \wedge\left(\left(a \otimes a_{f}\right) \vee\left(a_{+} \otimes 1\right)\right)\right) \vee g
$$

and

$$
g_{a}=\left(f \wedge\left(\left(a \otimes a_{g}\right) \vee\left(a_{+} \otimes 1\right)\right)\right) \vee g .
$$

Thus $f_{a} \equiv g_{a}\left(\theta_{f, g}\right)$. If $a$ is minimal in $J(A)$, then $a_{+}=0$, so that $g_{a}=g$. Note that $f_{\alpha}$ equals

$$
\begin{aligned}
\left(a \otimes a_{f}\right) & \bigvee \bigvee\left\{a^{\prime} \otimes a_{f}^{\prime} \mid a^{\prime}<a, a^{\prime} \in J(A)\right\} \\
& \bigvee \bigvee\left\{a^{\prime \prime} \otimes a_{g}^{\prime \prime} \mid a^{\prime \prime} \nless a, a^{\prime \prime} \in J(A)\right\}
\end{aligned}
$$

and $g_{a}$ equals

$$
\begin{aligned}
\left(a \otimes a_{g}\right) & \bigvee \bigvee\left\{a^{\prime} \otimes a_{f}^{\prime} \mid a^{\prime}<a, a^{\prime} \in J(A)\right\} \\
& \bigvee \bigvee\left\{a^{\prime \prime} \otimes a_{g}^{\prime \prime} \mid a^{\prime \prime} \nless a, a^{\prime \prime} \in J(A)\right\}
\end{aligned}
$$

which, in turn, equals $\bigvee\left\{f_{a^{\prime}} \mid a\right.$ covers $a^{\prime}$ in $\left.J(A)\right\}$. Hence by induction on the height of the poset $J(A), f_{a} \equiv g\left(\theta_{f, g}\right)$ for all $a \in J(A)$. Since $f=\bigvee\left\{f_{a} \mid a \in J(A)\right\}$, we obtain $f \equiv g\left(\theta_{f, g}\right)$.

THEOREM 3.6. Every principal congruence in $\operatorname{Con}_{L}(A \otimes B)$ can be represented as a finite join of congruences of the form

$$
\theta\left((a \otimes b) \vee\left(a_{+} \otimes 1\right),\left(a \otimes b_{+}\right) \vee\left(a_{+} \otimes 1\right)\right)
$$

where $a \in J(A)$ and $b>b_{+}$in $B$. 
We now fix $f=(a \otimes b) \vee\left(a_{+} \otimes 1\right)$ and $g=\left(a \otimes b_{+}\right) \vee\left(a_{+} \otimes 1\right)$ where $a \in$ $J(A)$ and $b>b_{+}$in $B$. We will show that

$$
\theta(f, g)=\theta\left(a, a_{+}\right)^{*} \wedge \theta\left(b, b_{+}\right)^{*}
$$

where $\theta\left(a, a_{+}\right)$and $\theta\left(b, b_{+}\right)$are the principal congruences in $A$ and $B$ generated by $\left(a, a_{+}\right)$and $\left(b, b_{+}\right)$, respectively.

LemMA 3.7. Let $a_{1}$ cover $a_{2}$ in A. Define $\rho: B \rightarrow A \otimes B$ by

$$
\rho\left(b^{\prime}\right)=\left(a_{1} \otimes b^{\prime}\right) \vee\left(a_{2} \otimes 1\right) \text {. }
$$

Then $\rho$ is an isomorphism onto $\left[a_{2} \otimes 1, a_{1} \otimes 1\right]$.

Proof. Clearly $\rho$ is a one-to-one join homomorphism. To see that $\rho$ preserves meets, it suffices to show that

$$
\left(\left(a_{1} \otimes b_{1}\right) \vee\left(a_{2} \otimes 1\right)\right) \wedge\left(\left(a_{1} \otimes b_{2}\right) \vee\left(a_{2} \otimes 1\right)\right) \leqslant\left(a_{1} \otimes\left(b_{1} \wedge b_{2}\right)\right) \vee\left(a_{2} \otimes 1\right)
$$

This readily follows from 2.3 and 2.4 . To show that $\rho$ is onto $\left[a_{2} \otimes 1, a_{1} \otimes 1\right]$, let $a_{2} \otimes 1 \leqslant h \leqslant a_{1} \otimes 1$. Then

$$
h=\bigvee\left\{\left(c_{j} \otimes d_{j}\right) \bigvee\left(a_{2} \otimes 1\right) \mid 1 \leqslant j \leqslant n\right\}
$$

which, by 2.1 , equals

$$
\bigvee\left\{\left(\left(c_{j} \vee a_{2}\right) \otimes d_{j}\right) \vee\left(a_{2} \otimes 1\right) \mid 1<j<n\right\},
$$

and so we may assume $c_{j} \geqslant a_{2}$. But $h \leqslant a_{1} \otimes 1$ implies that $c_{j} \leqslant a_{1}$; if $c_{j}<a_{1}$, then $c_{j}=a_{2}$ and so $\left(c_{j} \otimes d_{j}\right) \vee\left(a_{2} \otimes 1\right)=a_{2} \otimes 1$. Hence

$$
\begin{aligned}
h & =\left(\bigvee\left\{\left(a_{1} \otimes d_{j}\right) \vee\left(a_{2} \otimes 1\right) \mid 1 \leqslant j \leqslant m\right\}\right) \vee\left(a_{2} \otimes 1\right) \\
& =\left(a_{1} \otimes d\right) \vee\left(a_{2} \otimes 1\right)
\end{aligned}
$$

where $d=\bigvee\left\{d_{j} \mid 1 \leqslant j \leqslant m\right\}$. Thus $\rho$ is onto.

Lemma 3.8. Let $a \in J(A)$. For $\phi \in \operatorname{Con}_{L}(A), \psi \in \operatorname{Con}_{L}(B)$, and $b_{1} \neq b_{2}$ in $B$,

$$
\left(a \otimes b_{1}\right) \vee\left(a_{+} \otimes 1\right) \equiv\left(a \otimes b_{2}\right) \vee\left(a_{+} \otimes 1\right)\left(\phi^{*}\right)
$$

iff $a \equiv a_{+}(\phi)$, and

$$
\left(a \otimes b_{1}\right) \vee\left(a_{+} \otimes 1\right) \equiv\left(a \otimes b_{2}\right) \vee\left(a_{+} \otimes 1\right)\left(\psi^{*}\right)
$$

iff $b_{1} \equiv b_{2}(\psi)$.

Proof. By 3.1, $(A \otimes B) / \phi^{*} \simeq(A / \phi) \otimes B$. By 3.7, if $[a] \phi \neq\left[a_{+}\right] \phi$, then $B \simeq$ $\left[\left[a_{+}\right] \phi \otimes 1,[a] \phi \otimes 1\right]$. Thus the first claim follows. By 3.1, $(A \otimes B) / \psi^{*} \simeq A \otimes$ $(B / \psi)$. By 3.7,

$$
B / \psi \simeq\left[a_{+} \otimes[1] \psi, a \otimes[1] \psi\right]
$$

Thus the second claim follows.

Lemma 3.9. Let $a_{0}, a_{1}, a_{2} \in A$ with $a_{0} \leqslant a_{1}$ and $b_{0}, b_{1} \in B$. Then the following hold.

(i) $\left(\left(a_{1} \otimes b_{0}\right) \vee\left(a_{2} \otimes 1\right)\right) \bigvee\left(a_{1} \otimes b_{1}\right)=\left(a_{1} \otimes\left(b_{0} \vee b_{1}\right)\right) \vee\left(a_{0} \otimes 1\right)$,

(ii) $\left(\left(a_{1} \otimes b_{0}\right) \vee\left(a_{0} \otimes 1\right)\right) \wedge\left(\left(a_{1} \otimes b_{1}\right) \vee\left(a_{0} \otimes 1\right)\right)=\left(a_{1} \otimes\left(b_{0} \wedge b_{1}\right)\right) \vee$ $\left(a_{0} \otimes 1\right)$, 
(iii) $\left(\left(a_{1} \otimes b_{0}\right) \bigvee\left(a_{0} \otimes 1\right)\right) \bigvee\left(a_{2} \otimes 1\right)=\left(\left(a_{1} \vee a_{2}\right) \otimes b_{0}\right) \bigvee\left(\left(a_{0} \vee a_{1}\right) \otimes 1\right)$,

(iv) $\left(\left(a_{1} \otimes b_{0}\right) \vee\left(a_{0} \otimes 1\right)\right) \wedge\left(a_{2} \otimes 1\right)=\left(\left(a_{1} \wedge a_{2}\right) \otimes b_{0}\right) \vee\left(\left(a_{0} \wedge a_{2}\right) \otimes 1\right)$.

PRoof. These readily follow from 2.3 and 2.4 .

LEMMA 3.10. Let $a_{0}, a_{1}, a_{2}, a_{3} \in A$ with $a_{0} \leqslant a_{1}$ and $a_{2} \leqslant a_{3}$. If $a_{2} \equiv a_{3}\left(\theta\left(a_{0}, a_{1}\right)\right)$, then for any $b_{0} \leqslant b_{1}$ in $B$,

$$
\left(a_{3} \otimes b_{1}\right) \vee\left(a_{2} \otimes 1\right) \equiv\left(a_{3} \otimes b_{0}\right) \vee\left(a_{2} \otimes 1\right)
$$

modulo the congruence

$$
\theta\left(\left(a_{1} \otimes b_{1}\right) \bigvee\left(a_{0} \otimes 1\right),\left(a_{1} \otimes b_{0}\right) \bigvee\left(a_{0} \otimes 1\right)\right)
$$

Proof. Without loss of generality, we may assume that the quotient $a_{3} / a_{2}$ is weakly projective into $a_{1} / a_{0}$. Then in $A \otimes B$ the quotient

$$
\left(\left(a_{1} \otimes b_{1}\right) \vee\left(a_{0} \otimes 1\right)\right) /\left(\left(a_{1} \otimes b_{0}\right) \vee\left(a_{0} \otimes 1\right)\right)
$$

is weakly projective into the quotient

$$
\left(\left(a_{3} \otimes b_{1}\right) \vee\left(a_{2} \otimes 1\right)\right) /\left(\left(a_{3} \otimes b_{0}\right) \vee\left(a_{2} \otimes 1\right)\right)
$$

as follows: If at the $i$ th step in $A$ we go from $u_{i} / v_{i}$ to $\left(u_{i} \vee c_{i}\right) /\left(v_{i} \vee c_{i}\right)$, then in $A \otimes B$ we go from

$$
\left(\left(u_{i} \otimes b_{1}\right) \bigvee\left(v_{i} \otimes 1\right)\right) /\left(\left(u_{i} \otimes b_{0}\right) \vee\left(v_{i} \otimes 1\right)\right)
$$

to

$$
\frac{\left(\left(u_{i} \vee c_{i}\right) \otimes b_{1}\right) \vee\left(\left(v_{i} \vee c_{i}\right) \otimes 1\right)}{\left(\left(u_{i} \vee c_{i}\right) \otimes b_{0}\right) \vee\left(\left(v_{i} \vee c_{i}\right) \otimes 1\right)}
$$

by using 3.9(iii). We make analogous use of 3.9(iv) if the $i$ th step is a meet.

Lemma 3.11. Let $b_{0}, b_{1}, b_{2}, b_{3} \in B$ with $b_{0} \leqslant b_{1}$ and $b_{2} \leqslant b_{3}$. If $b_{3} \equiv b_{2}\left(\theta\left(b_{0}, b_{1}\right)\right)$, then for any $a_{0} \leqslant a_{1}$ in $A$,

$$
\left(a_{1} \otimes b_{3}\right) \vee\left(a_{0} \otimes 1\right) \equiv\left(a_{1} \otimes b_{2}\right) \vee\left(a_{0} \otimes 1\right)
$$

modulo the congruence

$$
\theta\left(\left(a_{1} \otimes b_{1}\right) \bigvee\left(a_{0} \otimes 1\right),\left(a_{1} \otimes b_{0}\right) \bigvee\left(a_{0} \otimes 1\right)\right) .
$$

Proof. The proof is similar to that of 3.10, using 3.9(i), (ii).

THEOREM 3.12. $\theta(f, g)=\theta\left(a, a_{+}\right)^{*} \wedge \theta\left(b, b_{+}\right)^{*}$.

Proof. By 3.5, $\theta(f, g) \leqslant \theta\left(a, a_{+}\right)^{*} \wedge \theta\left(b, b_{+}\right)^{*}$. For the reverse inclusion, let

$$
f^{\prime}=\left(a^{\prime} \otimes b^{\prime}\right) \bigvee\left(a_{+}^{\prime} \otimes 1\right), \quad g^{\prime}=\left(a^{\prime} \otimes b_{+}^{\prime}\right) \vee\left(a_{+}^{\prime} \otimes 1\right),
$$

where $a^{\prime}$ is join irreducible and $b>b_{+}^{\prime}$. Then we need only show that $\theta\left(f^{\prime}, g^{\prime}\right) \leqslant$ $\theta\left(a, a_{+}\right)^{*} \wedge \theta\left(b, b_{+}\right)^{*}$ implies that $\theta\left(f^{\prime}, g^{\prime}\right) \leqslant \theta(f, g)$. By 3.8, $\theta\left(f^{\prime}, g^{\prime}\right) \leqslant \theta\left(a, a_{+}\right)^{*}$ $\wedge \theta\left(b, b_{+}\right)^{*}$ implies that

$$
a^{\prime} \equiv a_{+}^{\prime}\left(\theta\left(a, a_{+}\right)\right) \quad \text { and } \quad b^{\prime} \equiv b_{+}^{\prime}\left(\theta\left(b, b_{+}\right)\right) .
$$

But then, by 3.10 and 3.11 ,

$$
f^{\prime} \equiv g^{\prime}\left(\theta\left(\left(a \otimes b^{\prime}\right) \vee\left(a_{+} \otimes 1\right),\left(a \otimes b_{+}^{\prime}\right) \vee\left(a_{+} \otimes 1\right)\right)\right)
$$


and

$$
\left(a \otimes b^{\prime}\right) \vee\left(a_{+} \otimes 1\right) \equiv\left(a \otimes b_{+}^{\prime}\right) \vee\left(a_{+} \otimes 1\right)(\theta(f, g))
$$

Hence $\theta\left(f^{\prime}, g^{\prime}\right) \leqslant \theta(f, g)$, proving the theorem.

LemmA 3.13. Let $a \in J(A)$ and let $b>b_{+}$in $B$; let $\phi \in \operatorname{Con}_{L}(A)$ and $\psi \in$ $\operatorname{Con}_{L}(B)$. Then

$$
\theta\left(a, a_{+}\right)^{*} \wedge \theta\left(b, b_{+}\right)^{*} \leqslant \phi^{*} \text { iff } \theta\left(a, a_{+}\right) \leqslant \phi
$$

and

$$
\theta\left(a, a_{+}\right)^{*} \wedge \theta\left(b, b_{+}\right)^{*} \leqslant \psi^{*} \text { iff } \theta\left(b, b_{+}\right) \leqslant \psi .
$$

Proof. If $\theta\left(a, a_{+}\right) \leqslant \phi$, then trivially $\theta\left(a, a_{+}\right)^{*} \wedge \theta\left(b, b_{+}\right)^{*}<\phi^{*}$, and if $\theta\left(b, b_{+}\right) \leqslant \psi$, then trivially $\theta\left(a, a_{+}\right)^{*} \wedge \theta\left(b, b_{+}\right)^{*} \leqslant \psi^{*}$. Suppose $\theta\left(a, a_{+}\right) \nless \phi$ and $\theta\left(b, b_{+}\right) \leqslant \psi$. By 3.12 ,

$$
\theta\left(a, a_{+}\right)^{*} \wedge \theta\left(b, b_{+}\right)^{*}=\theta\left((a \otimes b) \vee\left(a_{+} \otimes 1\right),\left(a \otimes b_{+}\right) \vee\left(a_{+} \otimes 1\right)\right)
$$

Hence it suffices to show that

$$
\theta\left((a \otimes b) \vee\left(a_{+} \otimes 1\right),\left(a \otimes b_{+}\right) \vee\left(a_{+} \otimes 1\right)\right)
$$

is not contained in either $\phi^{*}$ or $\psi^{*}$. By 3.1,

$(A \otimes B) / \phi^{*} \simeq(A / \phi) \otimes B$ and $(A \otimes B) / \psi^{*} \simeq A \otimes(B / \psi)$.

By 3.7,

$$
B / \psi \simeq\left[a_{+} \otimes[1] \psi, a \otimes[1] \psi\right]
$$

and since $[a] \phi \neq\left[a_{+}\right] \phi$,

$$
B \simeq\left[\left[a_{+}\right] \phi \otimes 1,[a] \phi \otimes 1\right]
$$

Thus in the first case,

$$
\left((a \otimes b) \vee\left(a_{+} \otimes 1\right),\left(a \otimes b_{+}\right) \vee\left(a_{+} \otimes 1\right)\right) \notin \psi^{*}
$$

and in the second,

$$
\left((a \otimes b) \vee\left(a_{+} \otimes 1\right),\left(a \otimes b_{+}\right) \vee\left(a_{+} \otimes 1\right)\right) \notin \phi^{*}
$$

THEOREM 3.14. The set

$$
C(A)=\left\{\phi^{*} \mid \phi \in \operatorname{Con}_{L}(A)\right\}
$$

is a sublattice of $\operatorname{Con}_{L}(A \otimes B)$ isomorphic to $\operatorname{Con}_{L}(A)$ and the set

$$
C(B)=\left\{\psi^{*} \mid \psi \in \operatorname{Con}_{L}(B)\right\}
$$

is a sublattice of $\operatorname{Con}_{L}(A \otimes B)$ isomorphic to $\operatorname{Con}_{L}(B)$.

Proof. It is clear that $\phi \rightarrow \phi^{*}$ and $\psi \rightarrow \psi^{*}$ are join isomorphisms. Thus it remains to show that $C(A)$ and $C(B)$ are closed under meets, that is, $\phi_{0}^{*} \wedge \phi_{1}^{*} \leqslant$ $\left(\phi_{0} \wedge \phi_{1}\right)^{*}$ and $\psi_{0}^{*} \wedge \psi_{1}^{*} \leqslant\left(\psi_{0} \wedge \psi_{1}\right)^{*}$. Note that $\phi_{0}^{*} \wedge \phi_{1}^{*}$ and $\psi_{0}^{*} \wedge \psi_{1}^{*}$ are lattice congruences. By 3.6 and 3.12, it suffices to show that for $a \in J(A)$ and $b>b_{+}$in $B$, if

$$
\left((a \otimes b) \vee\left(a_{+} \otimes 1\right),\left(a \otimes b_{+}\right) \vee\left(a_{+} \otimes 1\right)\right) \in \phi_{0}^{*} \wedge \phi_{1}^{*},
$$


then

$$
\left((a \otimes b) \vee\left(a_{+} \otimes 1\right),\left(a \otimes b_{+}\right) \vee\left(a_{+} \otimes 1\right)\right) \in\left(\phi_{0} \wedge \phi_{1}\right)^{*}
$$

and if

$$
\left((a \otimes b) \vee\left(a_{+} \otimes 1\right),\left(a \otimes b_{+}\right) \vee\left(a_{+} \otimes 1\right)\right) \in \psi_{0}^{*} \wedge \psi_{1}^{*}
$$

then

$$
\left((a \otimes b) \vee\left(a_{+} \otimes 1\right),\left(a \otimes b_{+}\right) \vee\left(a_{+} \otimes 1\right)\right) \in\left(\psi_{0} \wedge \psi_{1}\right)^{*}
$$

But these follow readily from 3.12 and 3.13 .

Let $D_{0,1}$ be the category of bounded distributive lattices with bound-preserving homomorphisms. For $C, D \in D_{0,1}$, let $C * D$ be the free product of $C$ and $D$ in $D_{0,1}$.

Lemma 3.15. Let $C, D \in D_{0,1}$. In $S_{0}$ form $C \otimes D$, the tensor product of $C$ and $D$, and in $D_{0,1}$ form $C * D$, the free product of $C$ and $D$. Then $C \otimes D \simeq C * D$.

Proof. Since $D_{0,1}$ is locally finite and direct limits commute with both tensor products and free products, we may assume that both $C$ and $D$ are finite. But then $J(C * D) \simeq J(C) \times J(D)$. Thus $C * D$ is the free join semilattice with zero generated by $J(C) \times J(D)$ (regarded as a partial join semilattice where joins are defined only for comparable elements). Therefore, we can find a join homomorphism from $C * D$ onto $C \otimes D$. On the other hand, $f: C \circ D \rightarrow C * D$ defined by $f(c, d)=c$ $\wedge d$ is a bimorphism since $C * D$ is distributive. Hence we can find a homomorphism from $C \otimes D$ onto $C * D$. Since $C \otimes D$ and $C * D$ are finite, they must be isomorphic semilattices, and hence isomorphic lattices.

THEOREM 3.16. The following isomorphism holds.

$$
\operatorname{Con}_{L}(A \otimes B) \simeq \operatorname{Con}_{L}(A) \otimes \operatorname{Con}_{L}(B) \text {. }
$$

Proof. Since the congruence lattice of a lattice is distributive, we invoke 3.15 and prove that $\operatorname{Con}_{L}(A \otimes B) \simeq \operatorname{Con}_{L}(A) * \operatorname{Con}_{L}(B)$. We use the solution to the word problem for free products of bounded distributive lattices (see G. Grätzer [6]). By 3.12, $C(A) \cup C(B)$ generates $\operatorname{Con}_{L}(A \otimes B)$. By 3.14, $C(A) \simeq \operatorname{Con}_{L}(A)$ and $C(B) \simeq \operatorname{Con}_{L}(B)$. Let $\phi_{0}, \phi_{1} \in \operatorname{Con}_{L}(A)$ and $\psi_{0}, \psi_{1} \in \operatorname{Con}_{L}(B)$. It suffices to show that if $\phi_{0}^{*} \wedge \psi_{0}^{*} \leqslant \phi_{1}^{*} \vee \psi_{1}^{*}$, then $\phi_{0}^{*} \leqslant \phi_{1}^{*}$ or $\psi_{0}^{*} \leqslant \psi_{1}^{*}$. Suppose $\phi_{0}^{*} \leqslant \phi_{1}^{*}$; thus there are $a_{1}$ covering $a_{0}$ in $A$ with $a_{1} \equiv a_{0}\left(\phi_{0}\right)$ but not $a_{1} \equiv a_{0}\left(\phi_{1}\right)$. By 3.7, $\left[a_{0} \otimes 1\right.$, $\left.a_{1} \otimes 1\right] \simeq B$. Moreover, $\phi_{0}^{*}=\imath$ on $\left[a_{0} \otimes 1, a_{1} \otimes 1\right]$ while $\phi_{1}^{*}=\omega$ on $\left[a_{0} \otimes 1\right.$, $\left.a_{1} \otimes 1\right]$. Hence on $\left[a_{0} \otimes 1, a_{1} \otimes 1\right]$ we have $\psi_{0}^{*}=\phi_{0}^{*} \wedge \psi_{0}^{*} \leqslant \phi_{1}^{*} \vee \psi_{1}^{*}$. But since $\left[a_{0} \otimes 1, a_{1} \otimes 1\right]$ is a convex sublattice of $A \otimes B, \phi_{1}^{*} \vee \psi_{1}^{*}=\psi_{1}^{*}$ on $\left[a_{0} \otimes 1, a_{1} \otimes\right.$ 1]. Because of the canonical isomorphism between $\left[a_{0} \otimes 1, a_{1} \otimes 1\right]$ and $B$, this means that $\psi_{0} \leqslant \psi_{1}$ and therefore $\psi_{0}^{*} \leqslant \psi_{1}^{*}$ on $A \otimes B$. This proves the theorem.

Lemma 3.17. Let $A, B \in S_{0}$, and let $A_{0}$ and $B_{0}$ be sublattices of $A$ and $B$, respectively. Then the canonical homomorphism from $A_{0} \otimes B_{0}$ to $A \otimes B$ is a lattice embedding. 
Proof. Let $a_{i} \in A_{0}, b_{i} \in B_{0}$ for $i=1, \ldots, n$, and let $a \in A, b \in B$. Then the solution to the word problem tells us that in $A \otimes B$,

$$
a \otimes b \leqslant \bigvee\left\{a_{i} \otimes b_{i} \mid 1 \leqslant i \leqslant n\right\}
$$

if and only if there are $a^{\prime} \in A_{0}, b^{\prime} \in B_{0}$ such that $a \otimes b \leqslant a^{\prime} \otimes b^{\prime}$ and

$$
a^{\prime} \otimes b^{\prime} \leqslant \bigvee\left\{a_{i} \otimes b_{i} \mid 1 \leqslant i \leqslant n\right\}
$$

in $A_{0} \otimes B_{0}$. Thus the canonical homomorphism is an embedding. Moreover, using 2.4 we see that the canonical homorphism also preserves meets.

Now we are ready to state and prove our main result.

Theorem 3.18. Let $A, B \in S_{0}$ be lattices; let $A$ be locally finite, and let $B$ be $A$-lower bounded. Then $\operatorname{Con}_{L}(A \otimes B) \simeq \operatorname{Con}_{L}(A) \otimes \operatorname{Con}_{L}(B)$.

Proof. By 3.17, $A \otimes B$ is the direct limit (as lattices) of the lattices $A_{f} \otimes B_{1}$, where $A_{f}$ ranges over the finite sublattices of $A$ and $B_{1}$ ranges over the sublattices of $B$ with unit. By 3.16,

$$
\operatorname{Con}_{L}\left(A_{f} \otimes B_{1}\right) \simeq \operatorname{Con}_{L}\left(A_{f}\right) \otimes \operatorname{Con}_{L}\left(B_{1}\right)
$$

But $\operatorname{Con}_{L}(A \otimes B)$ is the direct limit of the lattices $\operatorname{Con}_{L}\left(A_{f} \otimes B_{1}\right)$ where $A_{f}$ ranges over the finite sublattices of $A$ and $B_{1}$ ranges over the sublattices of $B$ with unit. Since direct limits and tensor products commute, our result follows.

4. Consequences of the main result. The following lemma is obvious.

LEMMA 4.1. Let $A$ and $B$ be bounded distributive lattices, each with a unique atom. Then $A * B$ has a unique atom.

Throughout this section we assume that $A, B \in S_{0}$ are lattices such that $A$ is locally finite and $B$ is $A$-lower bounded.

TheOREM 4.2. As a lattice, $A \otimes B$ is subdirectly irreducible iff both $A$ and $B$ are subdirectly irreducible lattrices.

Proof. Since $A$ is subdirectly irreducible iff $\operatorname{Con}_{L}(A)$ has a unique atom, the theorem follows from 4.1 and the main result (3.18).

Theorem 4.3. Let $A$ be a simple lattice; then $\operatorname{Con}_{L}(A \otimes B) \simeq \operatorname{Con}_{L}(B)$.

Proof. $A$ is simple iff $\operatorname{Con}_{L}(A) \simeq C_{2}$, and $C_{2} * D \simeq D$ for any $D \in D_{0,1}$.

Corollary 4.4. As a lattice, $A \otimes B$ is simple iff both $A$ and $B$ are simple lattices.

Corollary 4.5 (E. T. Schmidt [8]). For any $D \in B_{0,1}, \operatorname{Con}_{L}\left(M_{3}[D]\right) \simeq$ $\operatorname{Con}_{L}(D)$.

TheOREM 4.6. Let $\theta \in \operatorname{Con}_{L}(A \otimes B)$; then $(A \otimes B) / \theta$ is subdirectly irreducible iff $\theta=\phi^{*} \vee \psi^{*}$ where $A / \phi$ and $B / \psi$ are subdirectly irreducible.

Proof. $(A \otimes B) / \theta$ is subdirectly irreducible iff $\theta$ is completely meet irreducible. Since $\theta$ can be written as the meet of elements of the form $\phi^{*} \vee \psi^{*}$ (see [6]), and 
since $\theta$ is meet irreducible, $\theta=\phi^{*} \vee \psi^{*}$ for some $\phi \in \operatorname{Con}_{L}(A)$ and some $\psi \in$ $\operatorname{Con}_{L}(B)$. Thus $(A \otimes B) / \theta \simeq(A / \phi) \otimes(B / \psi)$ and so 4.2 tells us that $A / \phi$ and $B / \psi$ are subdirectly irreducible.

For a lattice $L$, let $D(L)$ be the maximal distributive quotient of $L$; let $\theta_{M}(L)$ (respectively, $\left.\theta_{D}(L)\right)$ be the kernel of the maximal modular (respectively, distributive) quotient of $L$.

THEOREM 4.7. The following isomorphism holds.

$$
D(A \otimes B) \simeq D(A) \otimes D(B) .
$$

Hence, the tensor product in $S_{0}$ of two distributive lattices with 0 is again a distributive lattice with 0 .

See G. Fraser [3].

Proof. Let $(A \otimes B) / \theta \simeq C_{2}$; then by $4.6, \theta=\phi^{*} \vee \psi^{*}$ where $A / \phi \simeq C_{2} \simeq$ $B / \psi$. From this the theorem readily follows.

Lemma 4.8. Let $A_{0}, A_{1}$ both be modular but not distributive; then $A_{0} \otimes A_{1}$ is not modular.

Proof. Let $z_{i}, a_{i}, b_{i}, c_{i}, u_{i} \in A_{i}$ form a sublattice isomorphic to $M_{3}$ (with $z_{i}$ the zero and $u_{i}$ the unit). We claim that

$$
\begin{gathered}
\left(z_{0} \otimes u_{1}\right) \vee\left(u_{0} \otimes z_{1}\right), \quad\left(a_{0} \otimes b_{1}\right) \vee\left(b_{0} \otimes a_{1}\right), \quad\left(a_{0} \otimes a_{1}\right) \vee\left(b_{0} \otimes b_{1}\right), \\
\left(a_{0} \otimes a_{1}\right) \vee\left(b_{0} \otimes b_{1}\right) \vee\left(c_{0} \otimes c_{1}\right), \quad\left(u_{0} \otimes u_{1}\right)
\end{gathered}
$$

form a nonmodular sublattice of $A_{0} \otimes A_{1}$. This follows readily from the solution to the word problem; the only nontrivial part is showing that

$$
\begin{aligned}
\left(\left(a_{0} \otimes b_{1}\right) \vee\left(b_{0} \otimes a_{1}\right)\right) & \wedge\left(\left(a_{0} \otimes a_{1}\right) \vee\left(b_{0} \otimes b_{1}\right) \vee\left(c_{0} \otimes c_{1}\right)\right) \\
& =\left(z_{0} \otimes u_{1}\right) \vee\left(u_{0} \otimes z_{1}\right) .
\end{aligned}
$$

But because $M_{3}$ is self-dual, we have

$$
(x \otimes y) \leqslant\left(a_{0} \otimes a_{1}\right) \vee\left(b_{0} \otimes b_{1}\right) \vee\left(c_{0} \otimes c_{1}\right)
$$

iff $(x \otimes y)$ is less than or equal to at least one of $\left(a_{0} \otimes a_{1}\right),\left(b_{0} \otimes b_{1}\right),\left(c_{0} \otimes c_{1}\right)$, $\left(z_{0} \otimes u_{1}\right),\left(u_{0} \otimes z_{1}\right)$. From this the result is straightforward.

THEOREM 4.9. The following equality holds.

$$
\theta_{M}(A \otimes B)=\left(\theta_{M}(A)^{*} \vee \theta_{D}(B)^{*}\right) \wedge\left(\theta_{D}(A)^{*} \vee \theta_{M}(B)^{*}\right)
$$

Proof. Let $(A \otimes B) / \theta$ be subdirectly irreducible and modular. By 4.6, $\theta=\phi^{*} \bigvee$ $\psi^{*}$ where $A / \phi$ and $B / \psi$ are subdirectly irreducible. By 4.8 and the main result, one of them is modular and the other is distributive. Hence

$$
\theta \geqslant\left(\theta_{M}(A)^{*} \vee \theta_{D}(B)^{*}\right) \wedge\left(\theta_{D}(A)^{*} \vee \theta_{M}(B)^{*}\right) \geqslant \theta_{M}(A \otimes B)
$$

But

$$
\theta_{M}(A \otimes B)=\bigwedge\{\theta \mid(A \otimes B) / \theta \text { is subdirectly irreducible and modular }\} ;
$$

this proves the theorem. 


\section{REFERENCES}

1. J. Anderson and N. Kimura, The tensor product of semilattices, Semigroup Forum 16 (1978), 83-88.

2. G. Fraser, The tensor product of distributive lattices, Proc. Edinburgh Math. Soc. (2) 20 (1976), 121-131.

3. __ The semilattice tensor product of distributive lattices, Trans. Amer. Math. Soc. 217 (1976), 183-194.

4. __ The tensor product of semilattices, Algebra Universalis 8 (1978), 1-3.

5. $178-184$.

6. G. Grätzer, Lattice theory: First concepts and distributive lattices, Freeman, San Francisco, Calif., 1971.

7. __ General lattice theory, Pure and Appl. Math. Ser., Academic Press, New York; Mathematische Reihe, Band 52, Birkhäuser Verlag, Basel; Akademie Verlag, Berlin, 1978.

8. E. T. Schmidt, Lattices generated by partial lattices, Lattice Theory (Proc. Colloq., Szeged, 1974), Colloq. Math. Soc. János Bolyai, Vol. 14, North-Holland, Amsterdam, 1974, pp. 343-353.

9. Z. Shmuely, The structure of Galois connections, Pacific J. Math. 54 (1974), 209-225.

Department of Mathematics and Astronomy, University of Manitoba, Winnipeg, Manitoba, CANADA R3T 2N2 